ORIGINAL PAPER

\title{
Clinicopathological characteristics of Segmental (IV-S) AND GLOBAL (IV-G) ACTIVE SUBCLASSES OF CLASS IV LUPUS NEPHRITIS: A COMPARATIVE STUDY
}

\author{
Matgorzata Wąrowska-Danilewicz ${ }^{1}$, Marian Danilewicz ${ }^{2}$
}

${ }^{1}$ Department of Nephropathology, Medical University of Lodz, Poland

${ }^{2}$ Department of Pathomorphology, Medical University of Lodz, Poland

\begin{abstract}
Class IV is the most common form of lupus nephritis (LN). This class is divided into diffuse segmental active subclass (IV-S/A) and diffuse global active subclass (IV-G/A). The purpose of the study was to compare the clinical data, and the incidence of glomerular active features in IV-S/A, and IV-G/A of LN. Of 34 patients with class IV LN, 25 were classified as subclass IV-G/A, and 9 patients were included in subclass IV-S/A. Nephrotic syndrome and hypertension were significantly more frequent in patients in the IV-G/A subclass, whereas hematuria and low grade proteinuria were significantly more frequent in patients in the IV-S/A subclass. Immunofluorescence findings revealed in group IV-S/A the presence of mesangial deposits and sparse deposits along the capillary wall. In contrast, an immunofluorescence study in subclass IV-G/A showed a preponderance of great subepithelial deposits. Fibrinoid necrosis of glomerular tufts was significantly more evident in subclass IV-S/A, whereas endocapillary hypercellularity, hyaline thrombi and wire loops were more frequent in subclass IV-G/A. The significant differences in clinical manifestation and in the incidence of histopathological active glomerular features between subclasses IV-S/A and IV-G/A of LN observed in our study may confirm the suggestion that these lesions have a different pathogenesis.
\end{abstract}

Key words: lupus nephritis, segmental lesions, global lesions.

\section{Introduction}

Renal disease is a common manifestation of systemic lupus erythematosus (SLE). Morphologically and clinically, lupus nephritis (LN) is a pleomorphic disease with the capacity to transform over time. Lupus nephritis is a prototype of chronic immune complex disease with activation of complement by classical and alternative pathways. The classification of SLE nephritis has evolved over the past years as more lesions were identified and defined. According to the International Society of Nephrology and Renal Pathology Society (ISN/RPS) classification of LN, class IV was subdivided into diffuse segmental LN (subclass IV-S) when $>50 \%$ of the involved glomeruli have segmental lesions, and diffuse global LN (subclass IV-G) when $>50 \%$ of the involved glomeruli have global lesions [1]. The division of class IV into two subcategories in the 2003 ISN/RPS classification was based on a pilot study observing a difference in renal outcome between segmental and global lesions in diffuse proliferative LN [2]. Glomerular active lesions include endocapillary hypercellularity, leukocyte infiltration, subendothelial hyaline deposits ("wire loops"), fibrinoid necrosis/karyorrhexis, and cellular crescents $[1,3]$. Renal biopsy has a major role in managing patients with SLE [4-8]. The distinction between subclass IV-S and IV-G may have important implications 
for differences in pathogenesis and outcomes. The data concerning outcomes in IV-S/A and IV-G/A patients are controversial, but in the majority of studies no significant differences in outcomes were detected $[2,4,9,10]$.

The aim of the study was to determine whether the clinical presentation and the incidence of active glomerular features differ in subclass IV-S/A and subclass IV-G/A of LN.

\section{Material and methods}

The material consisted of kidney biopsies obtained for diagnostic purposes from 34 adult patients with class IV of LN. All patients fulfilled the 1982 American Rheumatism Association criteria for the classification of SLE [11]. The diffuse glomerular lesions in IV class LN were reclassified using the ISN/RPS (2003) classification of glomerulonephritis in SLE [1]. Patients with lesions showing global lesions affect-

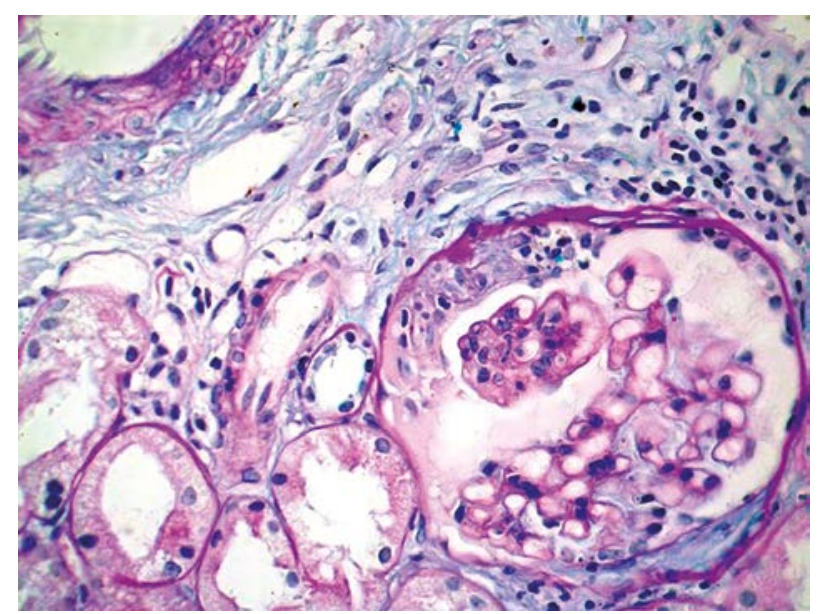

Fig. 1. Segmental endocapillary proliferation and cellular crescent. IV-S/A subclass of lupus nephritis. PAS staining, magnification $400 \times$

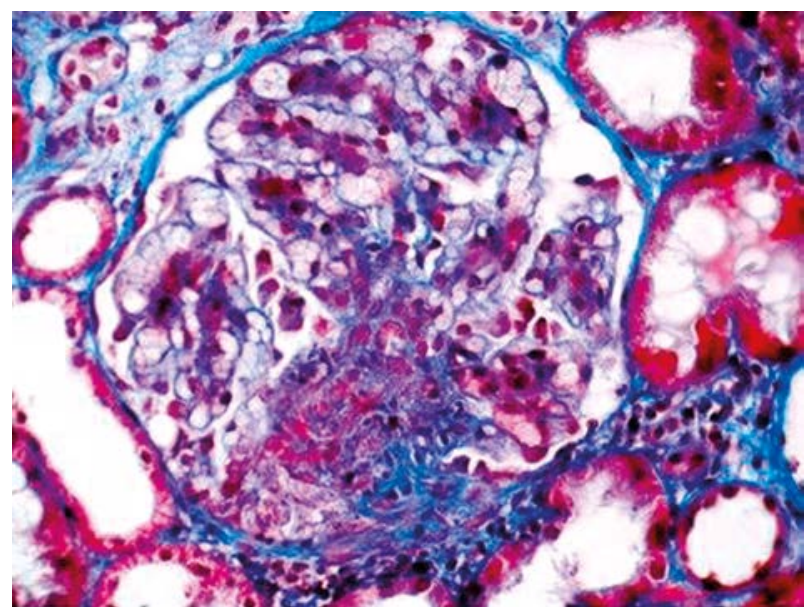

Fig. 2. Necrosis of glomerular capillary loops. IV-S/A subclass of lupus nephritis. Masson staining, magnification $400 \times$ ing $>50 \%$ of the glomerular tufts were classified as IV-G, whereas those with segmental lesions affecting $>50 \%$ of the glomerular tufts were classified as IV-S/A. Of the 34 patients, 9 had diffuse active segmental lesions (IV-S/A) and 25 had diffuse active global lesions (IV-G/A) of lupus nephritis. In group IV-S/A there were 8 females and 1 male, and the mean age was $31.2 \pm 9.3$ years. In group IV-G/A there were 19 females and 6 males, and the mean age was $29.5 \pm 8.2$ years. The following clinical parameters were evaluated at the time of each biopsy: the presence of hypertension, nephrotic syndrome, acute renal failure, chronic renal failure, proteinuria $<2 \mathrm{~g} /$ $24 \mathrm{~h}$, and hematuria.

Renal biopsy specimens were routinely processed by light microscopy, immunofluorescence, and in some cases by electron microscopy. The samples were embedded in paraffin and sectioned at $2 \mu \mathrm{m}$, followed by HE, Masson, periodic acid-Schiff, periodic acid-silver methenamine, and Congo red staining. For immunofluorescence study the samples were sectioned in frozen conditions, followed by staining for IgG, IgA, IgM, C3, C1q, kappa and lambda light chain. Criteria applied to the biopsy specimens were as follows: minimal number of 10 glomeruli for light microscopy, and 5 glomeruli for immunofluorescence study. In light microscopy the presence of endocapillary proliferation (Fig. 1), cellular crescents, necrosis of glomerular tufts (Fig. 2) (disruption of capillary wall, fibrinoid necrosis, karyorrhexis) hyaline thrombi, and wire loops (Fig. 3) were noted. In immunofluorescence findings localization of immune deposits, the intensity of immunoglobulin and components of complement staining and the class of immunoglobulin in deposits were evaluated. A semiquantitative assessment of the intensity of staining was given as: lack of staining $(0)$, mild $(+1)$, moderate $(+2)$, and strong $(+3)$.

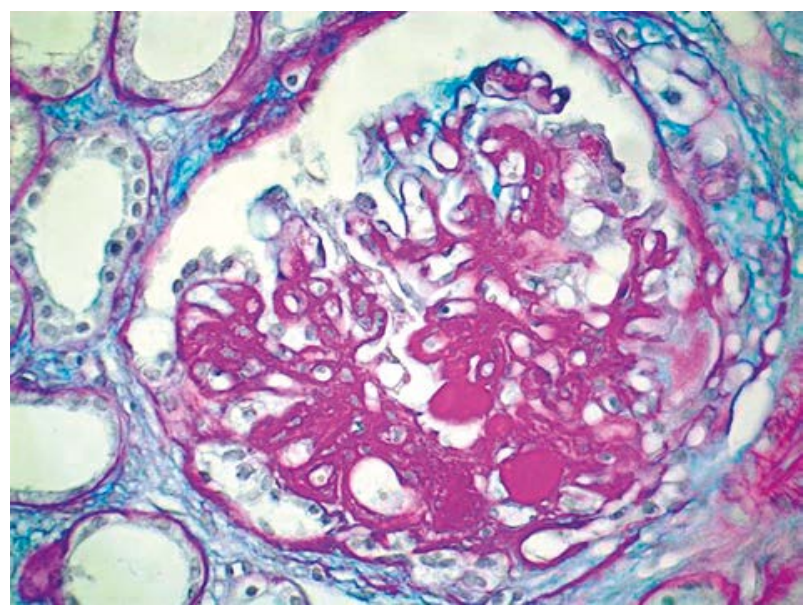

Fig. 3. Glomerulus with wire loops and hyaline thrombi. IV-G/A subclass of lupus nephritis. PAS staining, magnification $400 \times$ 


\section{Statistical analysis}

The percentage of each clinical, immunofluorescence and histological parameter was determined in the IVS/A and IV-G/A groups, and the differences between groups were assessed using the $\chi^{2}$ test. Results were considered statistically significant if $\mathrm{p}<0.05$.

\section{Results}

\section{Clinical data}

The results of clinical findings in patients with subclass IV-S/A and IV-G/A of LN are shown in Fig. 4. Statistical analysis revealed that SLE patients in the IV-S/A group had significantly more often hematuria $(\mathrm{p}<0.04)$ and proteinuria $<2 \mathrm{~g} / 24 \mathrm{~h}(\mathrm{p}<0.02)$ than patients in the IV-G/A group. Nephrotic syndrome and hypertension were more frequent in diffuse global active cases than in diffuse segmental active cases $(\mathrm{p}<0.05$, and $\mathrm{p}<0.04$, respectively). Statistical analysis did not reveal differences in the incidence of acute and chronic renal failure in patients with IV-S/A and IV-G/A LN.

\section{Immunofluorescence findings}

Immunofluorescence findings disclosed predominant mesangial deposits in the IV-S/A group ( $\mathrm{p}<$ $0.0001)$, and predominant deposits along the capillary loop in the IV-G/A group ( $\mathrm{p}<0.0001$ ). The intensity of $\operatorname{IgG}, \operatorname{IgA}$ and $\operatorname{IgM}$ staining was significantly higher in renal biopsies in IV-G/A patients than in the IV-S/A group. In the IV-S/A group immunofluorescence did not reveal strong $\operatorname{IgG}, \operatorname{IgA}$, and $\operatorname{IgM}$ staining. In most renal biopsies in the IVG/A group IgG staining was moderate or strong (IV-S/A vs. IV-G/A, p $<0.04$ ) (Fig. 5). In $89 \%$ of cases of subclass IV-S/A LN immunofluorescence did not reveal IgA staining $(\mathrm{p}<0.0001)$, and in this group only 11 cases showed mild $(+1)$ IgA staining $(\mathrm{p}<0.04)$ (Fig. 6). Most renal biopsies in the IV-S/A group did not reveal IgM staining $(p<0.0008)$, and only in $22 \%$ of biopsies in this group was mild IgM staining seen (Fig. 7). Immunofluorescence findings did not disclose significant differences in $\mathrm{C} 3$ or $\mathrm{Clq}$ staining between groups IV-S/A and IV-G/A LN.

\section{Light microscopy findings: glomerular active lesions}

Light microscopy findings revealed differences in the incidence of glomerular active lesions between groups IV-S/A and IV-G/A of LN (Fig. 8). Statistical analysis showed a high rate of fibrinoid necrosis in renal biopsies in the IV-S/A subclass of LN ( $\mathrm{p}<0.05)$. Endocapillary proliferation and hyaline thrombi were more often seen in renal biopsies in the IV-G/A subclass of
LN ( $\mathrm{p}<0.002, \mathrm{p}<0.04$, respectively). Wire loops were absent in all the biopsies in subclass IV-G/A LN.

Electron microscopy findings in the IV-G/A subclass revealed the presence of predominant large subendothelial deposits, whereas in the IV-S/A subclasses large deposits in the mesangium and scanty subendothelial deposits were seen.

\section{Discussion}

Our study disclosed significant differences in initial clinical presentation and morphologic features between segmental and global active subclasses of class IV LN. In patients with proliferative diffuse segmental active lesions (IV-S/A) LN pre-

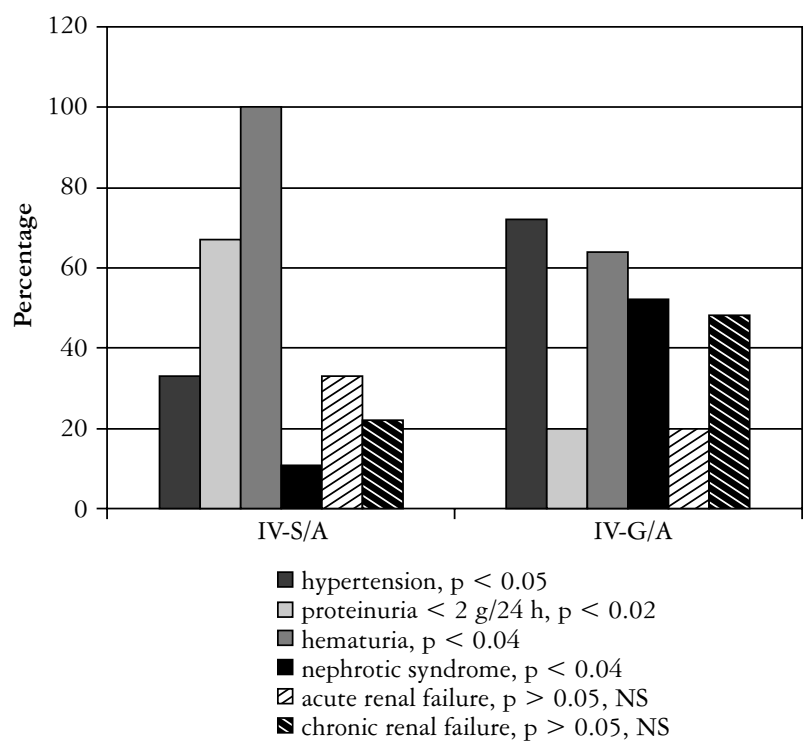

Fig. 4. Percentage of SLE patients presenting with hypertension, proteinuria $<2 \mathrm{~g} / 24 \mathrm{~h}$, hematuria, nephrotic syndrome, acute renal failure, and chronic renal failure in IV-S/A and IV-G/A groups

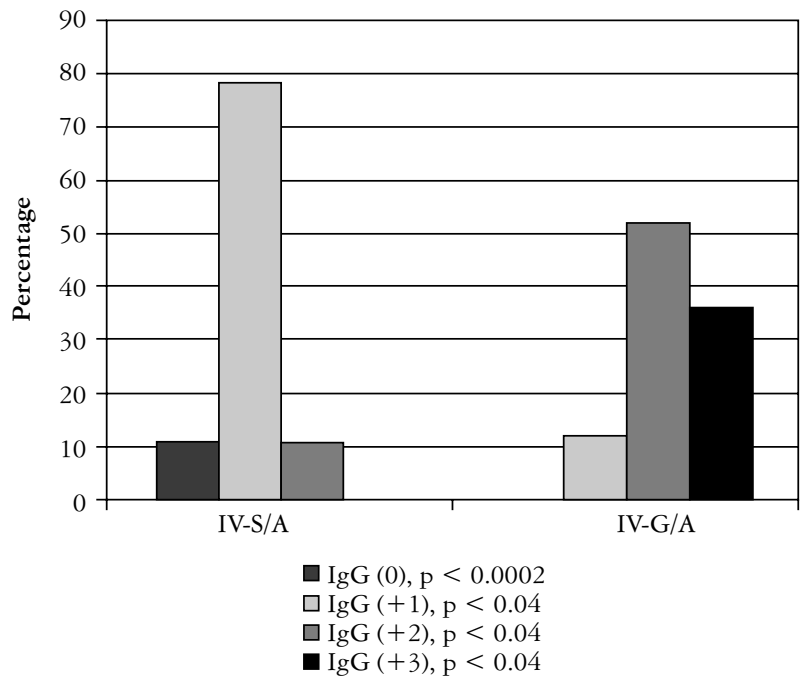

Fig. 5. Percentage of biopsies with $\operatorname{IgG}(0), \operatorname{IgG}(+1), \operatorname{IgG}$ $(+2)$, and $\operatorname{IgG}(+3)$ staining in IV-S/A and IV-G/A groups 


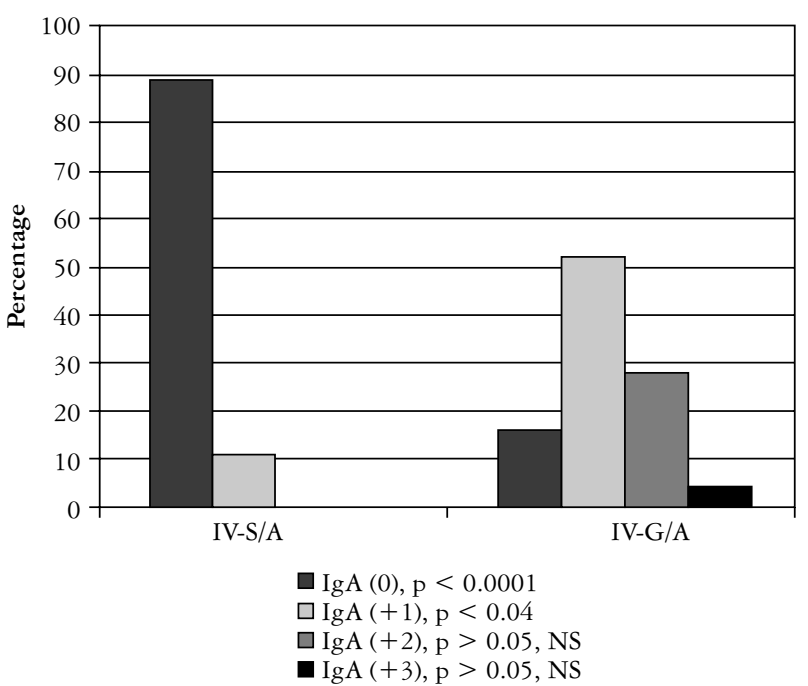

Fig. 6. Percentage of biopsies with $\operatorname{IgA}(0), \operatorname{IgA}(+1), \operatorname{IgA}$ $(+2)$, and $\operatorname{IgA}(+3)$ staining in IV-S/A and IV-G/A groups

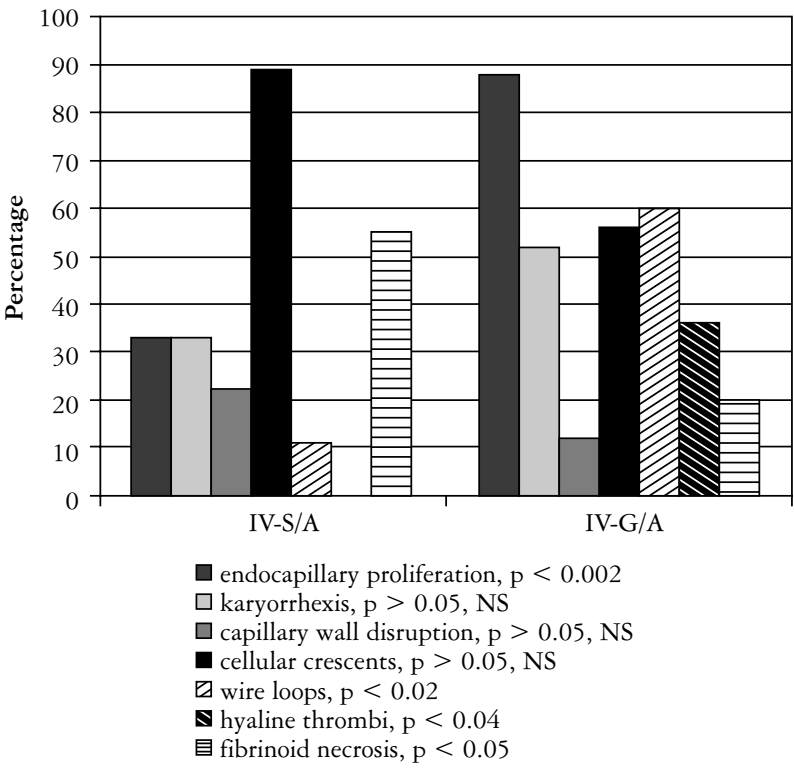

Fig. 8. Percentage of biopsies with endocapillary proliferation, karyorrhexis, capillary wall disruption, cellular crescents, wire loops, hyaline thrombi, and fibrinoid necrosis in IV-S/A and IV-G/A groups

ponderance of hematuria and proteinuria $<2 \mathrm{~g} /$ $24 \mathrm{~h}$ was seen, whereas in patients with proliferative diffuse global active features (IV-G/A) more often nephrotic syndrome and hypertension were detected. These observations are in concordance with others [3, 4, 10, 12-14]. Literature data showed that patients with IV-G lesions had worse proteinuria [3]. Monova et al. [13] disclosed in IV-G group patients significantly greater proteinuria, and diastolic blood pressure than in group IV-S of LN. Grootscholten et al. [10] observed that patients with IV-S features less often than with IV-G presented hypertension. In a large cohort of Chinese patients proteinuria was milder in IV-S cases than in IV-G [14]. However, in

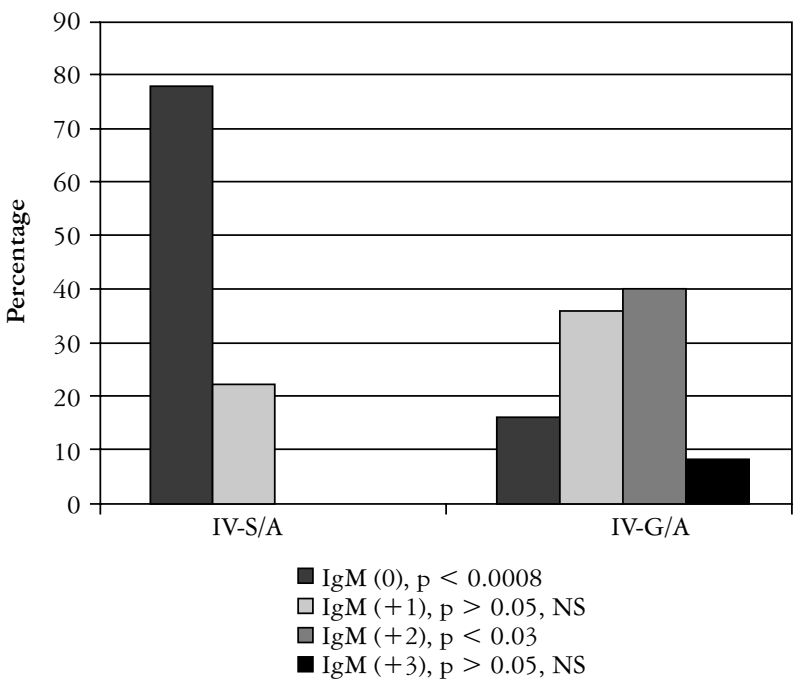

Fig. 7. Percentage of biopsies with $\operatorname{Ig} M(0), \operatorname{IgM}(+1), \operatorname{IgM}$ $(+2)$, and $\operatorname{IgM}(+3)$ staining in IV-S/A and IV-G/A groups

the study of Mittal et al. [15] significant proteinuria was similar in both IV-S and IV-G groups.

Our results demonstrated important differences in immunofluorescence findings between proliferative diffuse segmental active and proliferative diffuse global features of IV class LN. Peripheral capillary immune deposits were less prominent in the IV-S/A group than in IV-G/A cases. Moreover, the intensity of $\operatorname{IgG}, \operatorname{IgA}$ and $\operatorname{IgM}$ immunostaining was lower in segmental lesions than in global lesions. This finding may indicate a distinct immunopathogenesis that could require a different therapeutic protocol. Discrepancies in immunofluorescence findings in IV-S and IV-G subclasses of class IV LN were observed by others $[3,16]$. It is speculated that class IV-G lesions behave as an immune complex disease, whereas immunopathogenesis of segmental necrotizing features in the IV-S group may be similar to pauci-immune glomerulonephritis seronegative for ANCA. Proliferative and crescentic forms of vasculitis are associated with the delay-type hypersensitivity response in experimental models and could explain the capillary wall disruption seen in pauci-immune lupus lesions [17]. The delay-type hypersensitivity response may be initiated by granulocyte macrophage colony-stimulating factor induction of $\mathrm{MHC}$ II expression on macrophages leading to the promotion of the cytokine response of $\mathrm{T}$ cells towards T helper 1 cells [18]. The study of Schwartz et al. [19] supports the notion that two immunopathological mechanisms are concurrently active in severe LN. The focal segmental proliferative and necrotizing lesions that characterize severe segmental LN are often unassociated with peripheral capillary loop immune aggregate deposition. They are morphologically different from the endocapillary proliferation of diffuse global lupus glomerulonephritis, which appears to be mediated by a mechanism involving capillary wall 
immune aggregates [20]. The hypothesis of distinct immunopathogenesis in both subclasses of IV class LN may be substantiated by more frequent fibrinoid necrosis of glomerular tufts observed in renal tissue in patients of the IV-S/A group. Moreover, our results showed other striking differences in light microscopy findings between IV-S/A and IV-G/A subclasses. In proliferative global cases, endocapillary proliferation, hyaline thrombi, and wire loops were observed more often than in segmental active cases. An immunofluorescence study revealed that class IV-G/A exhibits larger amounts of immunoglobulin deposition in the capillary wall; thus it is clear that hyaline thrombi and wire loops are more common in this subclass of LN.

Literature data showed that despite the differences in clinical manifestation and histopathological findings observed in both subclasses of IV class LN, no significant difference was detected in outcomes in these groups [15]. The study of Haring et al. [2] revealed that the rate of doubling of serum creatinine concentration or of ESRD (end-stage renal disease) did not differ between patients with class IV-S and those with class IV-G LN. Kim et al. [8] in a short-term follow-up study found a better response to cyclophosphamide treatment in LN class IV-S than in IV-G. Vandepapeliere et al. [21] studied the prognosis of proliferative $\mathrm{LN}$ and concluded that the presence of even mild chronic lesions on baseline biopsy was clearly predictive of late renal outcome. Gao et al. [12] explored the characteristics and the correlation factors of transformation in subclasses of class IV lupus nephritis in patients with class IV LN after 6 months of induction treatment. Class IV-S showed a higher rate of transformation to class II than class IV-G. The recent study of Pagni et al. [22] stressed the importance of the distinction between class III, IV-S and IV-G. These authors concluded that the histopathological data suggest that morphological differences between segmental and global forms do exist, possibly due to different pathogenetic mechanisms.

In summary, the differences in the initial clinical presentation, immunofluorescence findings and $\mathrm{mi}$ croscopic active features in IV-S/A and IV-G/A LN revealed in our study confirm the suggestion that these lesions may have a different pathogenesis. Further studies should be conducted to clarify whether morphological and clinical differences between IV-S/A and IV-G/A LN are important for predicting the course of disease and response to therapy.

This work was supported by grant of Medical University of Lodz 503/6-038-01.

\section{References}

1. Weening JJ, D'Agati VD, Schwartz MM, et al. The classification of glomerulonephritis in systemic lupus erythematosus revisited. J Am Soc Nephrol 2004; 15: 241-250.

2. Haring CM, Rietveld A, van der Brand JA, et al. Segmental and global subclasses of class IV lupus nephritis have similar renal outcomes. J Am Soc Nephrol 2012; 23: 149-154.

3. Hill GS, Delahouse M, Nochy D, et al. Clas IV-s versus class IV-G lupus nephritis: clinical and morphologic differences suggesting different pathogenesis. Kidney Int 2005; 68: 22882207.

4. Mittal B, Rennke H, Singh A. The role of kidney biopsy in management of lupus nephritis. Curr Opin Nephrol Hypertens 2005; 14: 1-8.

5. Chang WY, Kim M-K, Lee HS. Predictors of renal outcome in diffuse proliferative lupus nephropathy: data from repeat renal biopsy. Nephrol Dial Transplant 2000; 15: 1604-1608.

6. Behara VY, Whittier WL, Korbet SM. Pathogenetic features of severe segmental lupus nephritis. Nephrol Dial Transplant 2010; 25: 153-159.

7. Schwartz MM, Korbet SM, Lewis EJ. The prognosis and pathogenesis of severe lupus glomerulonephritis. Nephrol Dial Transplant 2007; 23: 1298-1306.

8. Kim YG, Kim HW, Cho YM. The difference between lupus nephritis class IV-G and IV-S in Koreans: focus on the response to cyclophosphamide induction treatment. Rheumatology 2008; 47: 311-314.

9. Yokoyama H, Wada T, Hara A, et al. The outcome and a new ISN/RPS 2003 classification of lupus nephritis in Japanese. Kidney Int 2004; 66: 2382-2388.

10. Grootscholten C, Bajema IM, Florquin S, et al. Interobserver agreement of scoring of histopathological characteristic and classification of lupus nephritis. Nephrol Dial Transplant 2007; 23: 223-230.

11. Tan EM, Cohen AS, Fries JF, et al. The 1982 revised criteria for the classification of systemic lupus erythematosus. Arthritis Rheum 1982; 25: 1271-1277.

12. Gao JJ, Cai G, Liu S, et al. Characteristics and influency factors of pathologic transformation in the subclasses of class IV lupus nephritis. Rheumatol Int 2011; 32: 1751-1759.

13. Monova D, Monov S, Todorov T. Clinical and Morphologic Differences between Class IV-S and Class IV-G Lupus Nephritis, ISRN Immunology 2011; Article ID 502578, 5 pages, http://dx.doi.org/10.5402/2011/502578.

14. Yu F, Tan Y, Wu L-H. Class IV-G and IV-S lupus nephritis in Chinese patients: a large cohort study from a single center. Lupus 2009; 18: 1073-1081.

15. Mittal B, Hurwitz S, Rennke H, et al. New subcategories of class IV lupus nephritis: Are there clinical, histologic, and outcome difference? Am J Kid Dis 2004; 44: 1050-1059.

16. Yao CW, Kim MK, Lee HS. Predictors of renal outcome in diffuse proliferative lupus nephropathy: data from repeat renal biopsy. Nephrol Dial Transplant 2000; 15: 1604-1608.

17. Cunningham MA, Huang XR, Dowling JP, et al. Prominence of cell-mediated immunity effectors in "pauci-immune" glomerulonephritis. J Am Soc Nephrol 1999; 10: 499-506.

18. Timoshanco JR, Kitching AR, Semple TJ, et al. Granulocyte macrophage colony-stimulating factor expression by both renal parenchymal and immune cell mediates murine crescentic glomerulonephritis. J Am Soc Nephrol 2005; 16: 2646-2656.

19. Schwartz MM, Korbet SM, Katz RS, et al. Evidence of concurrent immunopathological mechanism determining the pathology of severe lupus nephritis. Lupus 2009; 18: 149-158.

20. Najafi CC, Korbet SM, Lewis EJ, et al. Nephritis collaborative study group: significance of histologic patterns of glomerular injury upon long-term prognosis in severe lupus glomerulonephritis. Kidney Int 2001; 59: 2156-2163. 
21. Vandepapeliere J, Aydin S, Cosyns J-P, et al. Prognosis of proliferative lupus nephritis subsets in the Louvain lupus nephritis inception cohort. Lupus 2014; 23: 159-165.

22. Pagni F, Galimberti S, Goffredo P, et al. The value of repeat biopsy in the management of lupus nephritis: an international multicentre study in a large cohort of patients. Nephrol Dial Transplant 2013; 28: 3014-3023.

\section{Address for correspondence}

Prof. Małgorzata Wagrowska-Danilewicz MD, PhD

Department of Nephropathology

Medical University of Lodz

Pomorska 251

92-216 Łódź, Poland

e-mail: malgorzata.wagrowska-danilewicz@umed.lodz.pl 